\title{
ON HARMONIC FUNCTIONS WITH INTEGRABLE MAXIMUM MODULUS
}

\author{
J. G. CLUNIE and P. J. RIPPON
}

\section{Introduction}

In [4] Hayman and Korenblum consider an extension of the Riesz-Herglotz formula. They consider functions

$$
g(z)=\sum_{1}^{\infty} a_{n} z^{n}=u+i v
$$

that are analytic in $U=\{|z|<1\}$ and investigate conditions on $u$ so that $g(z)$ can be represented as

$$
g(z)=\frac{i}{\pi} \int_{0}^{2 \pi} \frac{z e^{i t} \mu(t) d t}{\left(e^{i t}-z\right)^{2}} \quad(|z|<1),
$$

where $\mu(t)$ measurable and $\|\mu\|_{\infty}<\infty$. The necessary and sufficient conditions for such a representation with $\|\mu\|_{\infty} \leqq M$ are shown to be:

(i) If

then

$$
I\left(r, \varphi_{1}, \varphi_{2}\right)=\int_{\varphi_{1}}^{\varphi_{2}} u\left(r e^{i t}\right) d t,
$$

$$
\left|I\left(r, \varphi_{1}, \varphi_{2}\right)\right| \leqq 2 M \quad\left(0 \leqq r<1,-\infty<\varphi_{1}<\varphi_{2}<+\infty\right) .
$$

(ii) If the real constants $\varphi_{1}, \mu\left(\varphi_{1}\right)$ are suitably chosen then

$$
I\left(r, \varphi_{1}, \varphi_{2}\right) \rightarrow \mu\left(\varphi_{2}\right)-\mu\left(\varphi_{1}\right) \quad(r \rightarrow 1)
$$

for almost all fixed $\varphi_{2}$, where $\left|\mu\left(\varphi_{j}\right)\right| \leqq M(j=1,2)$.

It was also shown by Hayman and Korenblum in [4] that if $0<k(r) \nearrow$ in $0 \leqq r<1$ and

$$
u\left(r e^{i \theta}\right) \leqq k(r) \quad(0 \leqq r<1, \quad 0 \leqq \theta \leqq 2 \pi),
$$

where $u(z)$ is defined by (1.1), then the

$$
\int_{0}^{1} \sqrt{\frac{k(r)}{1-r}} d r<\infty
$$


implies that $g(z)$ has a representation (1.2) with $\|\mu\|_{\infty}<\infty$. On the other hand if the above integral diverges, then $g(z)$ exists as in (1.1), and (1.3) is satisfied, but $g(z)$ cannot be represented as in (1.2) with $\|\mu\|_{\infty}<\infty$.

In [5] Korenblum poses the problem of finding results analogous to the above when (1.3) is replaced by the two-sided condition

$$
\left|u\left(r e^{i \theta}\right)\right| \leqq k(r) \quad(0 \leqq r<1,0 \leqq \theta \leqq 2 \pi) .
$$

We shall show that for this problem one is led to consider $\int_{0}^{1} k(r) d r$ in place of $\int_{0}^{1}(k(r) /(1-r))^{1 / 2} d r$ and that if quite a weak regularity condition for $k(r)$ is satisfied then the former integral has the same significance for the two-sided condition (1.4) as the latter has for the one-sided condition (1.3). It is perhaps of interest to note that such a regularity condition is included in Korenblum's problem which appeared before the paper of Hayman and Korenblum.

Theorem 1. Suppose that (1.4) is satisfied, where $0<k(r) \nearrow$ in $0 \leqq r<1$ and $u(z)$ is defined by (1.1). If

then

$$
\int_{0}^{1} k(r) d r=J<\infty
$$

$$
\left|I\left(r, \varphi_{1}, \varphi_{2}\right)\right| \leqq 128 \mathrm{~J}\left(0 \leqq r<1,-\infty<\varphi_{1}<\varphi_{2}<+\infty\right)
$$

and there is a real-valued function $\mu$ on $(-\infty, \infty)$ satisfying

and

$$
|\mu(\varphi)| \leqq 64 \mathrm{~J} \quad(-\infty<\varphi<\infty)
$$

such that

$$
|\mu(\varphi+s)-\mu(\varphi)| \leqq 100 \int_{1-s}^{1} k(t) d t \quad\left(-\infty<\varphi<\infty, 0<s \leqq \frac{1}{2}\right)
$$

$$
I\left(r, \varphi_{1}, \varphi_{2}\right) \rightarrow \mu\left(\varphi_{2}\right)-\mu\left(\varphi_{1}\right) \quad(r \rightarrow 1)
$$

for $-\infty<\varphi_{1}<\varphi_{2}<+\infty$. Hence $g(z)$ has a representation (1.2), with $\|\mu\|_{\infty} \leqq 64 \mathrm{~J}$.

Remark. Finbarr Holland and Brian Twomey pointed out to us that this is only a slight generalisation of [2, Theorem 5.7]).

Theorem 2. Let $k(r) \nearrow$ in $0 \leqq r<1$ with $k(0)=1$ and $\int_{0}^{1} k(r) d r=\infty$. If, for some positive integer $n,(1-r)^{n} k(r) \backslash$ in $0 \leqq r<1$, then there exists $g=u+i v$ as in (1.1) with (1.4) satisfied, such that

$$
I(r, 0, \pi) \rightarrow \infty \quad(r \rightarrow 1) .
$$

Hence the necessary condition (i) of Hayman and Korenblum is not satisfied.

However, we shall show that in the absence of some regularity condition as occurs in Theorem 2 the two-sided result given in Theorem 1 is not best possible 
in the sense that the corresponding one-sided result of Hayman and Korenblum is best possible.

Theorem 3. There exists $0<k(r) / \infty$ in $0 \leqq r<1$ with $\int_{0}^{1} k(r) d r=\infty$ such that if $g=u+i v$ as in (1.1) and (1.4) is satisfied, then $g(z)$ has a representation (1.2) with $\|\mu\|_{\infty}<\infty$.

\section{Proof of Theorem 1}

If $g(z)=u+i v$ as in (1.1) then from Green's formula (or, see [4], proof of Theorem 1) we get

$$
I\left(r, \varphi_{1}, \varphi_{2}\right)=\int_{0}^{r} v\left(\varrho e^{i \varphi_{2}}\right) \frac{d \varrho}{\varrho}-\int_{0}^{r} v\left(\varrho e^{i \varphi_{1}}\right) \frac{d \varrho}{\varrho}
$$

for $0 \leqq r<1$ and $-\infty<\varphi_{1}<\varphi_{2}<\infty$. We now show that, for $-\infty<\varphi<\infty$,

$$
\int_{0}^{1}\left|v\left(\varrho e^{i \varphi}\right)\right| \frac{d \varrho}{\varrho} \leqq 64 \mathrm{~J}
$$

and if we then put

$$
\mu(\varphi)=\int_{0}^{1} v\left(\varrho e^{i \varphi}\right) \frac{d \varrho}{\varrho} \quad(-\infty<\varphi<\infty)
$$

the conclusions of Theorem 1, apart from (1.5), follow immediately.

To prove (2.2) we follow [2, Theorem 5.7] and set $\varrho=(1+r) / 2$ so that

$$
g(z)=\frac{1}{2 \pi} \int_{0}^{2 \pi} \frac{\varrho e^{i \varphi}+z}{\varrho e^{i \varphi}-z} u\left(\varrho e^{i \varphi}\right) d \varphi \quad(|z|=r<1) .
$$

Then, for $0 \leqq \theta \leqq 2 \pi$,

$$
\left|g^{\prime}\left(r e^{i \theta}\right)\right| \leqq \frac{1}{\pi} \int_{0}^{2 \pi} \frac{\left|u\left(\varrho e^{i(\theta+\varphi)}\right)\right|}{\varrho^{2}-2 \varrho r \cos \varphi+r^{2}} d \varphi \leqq \frac{1}{\pi} \int \frac{k(\varrho)}{\varrho^{2}-2 \varrho r \cos \varphi+r^{2}} d \varphi=\frac{2 k(\varrho)}{\varrho^{2}-r^{2}}
$$

so that

$$
\left|g^{\prime}\left(r e^{i \theta}\right)\right| \leqq \frac{8 k(\varrho)}{1-r}
$$

Thus, if

$$
M(r)=\max _{|z|=r}|g(z)|,
$$

then

$$
\int_{0}^{1} M(r) d r \leqq 8 \int_{0}^{1} d r \int_{0}^{r} \frac{k((1+t) / 2)}{1-t} d t=8 \int_{0}^{1} k\left(\frac{1+t}{2}\right) d t
$$

so that

$$
\int_{0}^{\mathbf{1}} M(r) d r \leqq 16 \mathrm{~J}
$$


By the maximum principle,

$$
\left|\frac{g(z)}{z}\right| \leqq 2 M\left(\frac{1}{2}\right) \quad\left(|z| \leqq \frac{1}{2}\right)
$$

and so, for $-\infty<\varphi<\infty$,

$$
\int_{0}^{1}\left|v\left(r e^{i \varphi}\right)\right| \frac{d r}{r} \leqq M\left(\frac{1}{2}\right)+2 \int_{1 / 2}^{1} M(r) d r \leqq 4 \int_{1 / 2}^{1} M(r) d r \leqq 64 \mathrm{~J}
$$

as required.

To prove (1.5) first note that from (2.1) and (2.3) we obtain

$$
\begin{gathered}
\mu(\varphi+s)-\mu(\varphi)=\int_{0}^{1}\left[v\left(t e^{i(\varphi+s)}\right)-v\left(t e^{i \varphi}\right)\right] \frac{d t}{t} \\
=I(r, \varphi, \varphi+s)+\int_{r}^{1}\left[v\left(t e^{i(\varphi+s)}\right)-v\left(t e^{i \varphi}\right)\right] \frac{d t}{t} \quad(0 \leqq r<1) .
\end{gathered}
$$

If $0<r<R<1$ and $-\infty<\varphi<\infty$,

$$
\int_{r}^{R} v\left(t e^{i \varphi}\right) \frac{d t}{t}=\left[v\left(t e^{i \varphi}\right) \log t\right]_{r}^{R}-\int_{r}^{R} \frac{\partial v\left(t e^{i \varphi}\right)}{\partial t} \log t d t
$$

and, since $v\left(t e^{i \varphi}\right)=o(1 /(1-t))(t \rightarrow 1)$ by (2.5), it follows that

$$
\begin{gathered}
\mu(\varphi+s)-\mu(\varphi)=I(r, \varphi, \varphi+s)-\left[v\left(r e^{i(\varphi+s)}\right)-v\left(r e^{i \varphi}\right)\right] \log r \\
-\int_{r}^{1}\left[\frac{\partial v\left(t e^{i(\varphi+s)}\right)}{\partial t}-\frac{\partial v\left(t e^{i \varphi}\right)}{\partial t}\right] \log t d t .
\end{gathered}
$$

Take $r=1-s$ with $1 / 2 \leqq r<1$, and then

Also, by (2.4),

$$
|I(r, \varphi, \varphi+s)| \leqq \int_{\varphi}^{\varphi+s}\left|u\left(r e^{i \theta}\right)\right| d \theta \leqq s k(r) \leqq \int_{1-s}^{1} k(t) d t .
$$

$\left|\left[v\left(r e^{i(\varphi+s)}\right)-v\left(r e^{i \varphi}\right)\right] \log r\right| \leqq 2(1-r) \int_{\varphi}^{\varphi+s}\left|\frac{\partial v\left(r e^{i \theta}\right)}{\partial \theta}\right| d \theta \leqq 16 s k\left(\frac{1+r}{2}\right) \leqq 32 \int_{-s}^{1} k(t) d t$.

In the same way, for $-\infty<\varphi<\infty$,

$$
\left|\int_{r}^{1} \frac{\partial v\left(t e^{i \varphi}\right)}{\partial t} \log t d t\right| \leqq \int_{r}^{1}\left|g^{\prime}\left(t e^{i \varphi}\right)\right| \log \frac{1}{t} d t \leqq 16 \int_{r}^{1} k\left(\frac{1+t}{2}\right) d t \leqq 32 \int_{1}^{1} k(\varrho) d \varrho .
$$

Using the above estimates in (2.6) we obtain (1.5). 


\section{Proof of Theorem 2}

Assume that the hypotheses of Theorem 2 are satisfied. Define $\varepsilon_{p}\left(0<\varepsilon_{p} \leqq 1\right)$ by

$$
k\left(1-\varepsilon_{p}\right)=p \quad(p=1,2, \ldots) .
$$

Then $\varepsilon_{p}$ decreases (strictly) to 0 as $p$ increases to $\infty$ and

$$
\sum_{p=1}^{\infty} \varepsilon_{p}=\infty
$$

follows from $\int_{0}^{1} k(r) d r=\infty$. Now put

$$
\lambda(r)=\sum_{p=1}^{\infty} r^{1 / \varepsilon_{p}} \quad(0 \leqq r<1) .
$$

If $1-\varepsilon_{q-1} \leqq r<1-\varepsilon_{q}, q \geqq 2$ and $K$ is a fixed positive integer, then

$$
\lambda(r) \leqq K q+\sum_{p=K q+1}^{\infty}\left(1-\varepsilon_{q}\right)^{1 / \varepsilon_{p}} \leqq K q+\sum_{p=K q+1}^{\infty} \exp \left(-\frac{\varepsilon_{q}}{\varepsilon_{p}}\right) .
$$

From the behaviour of $(1-r)^{n} k(r)$ and (3.1) we have

so that

$$
p \varepsilon_{p}^{n} \leqq q \varepsilon_{q}^{n} \quad(p \geqq q)
$$

$$
\frac{\varepsilon_{q}}{\varepsilon_{p}} \geqq\left(\frac{p}{q}\right)^{1 / n} \geqq 2 \log \frac{p}{q} \quad(p \geqq K q),
$$

if $K=K(n)$ is large enough. Hence for $1-\varepsilon_{q-1} \leqq r<1-\varepsilon_{q}$ and $q \geqq 2$,

$$
\lambda(r) \leqq K q+q^{2} \sum_{p=K q+1}^{\infty} p^{-2} \leqq(K+1) q \leqq 2(K+1) k\left(1-\varepsilon_{q-1}\right) \leqq 2(K+1) k(r),
$$

Let $n_{p}$ be the least odd integer exceeding $1 / \varepsilon_{p}$ and suppose that $\sum_{1}^{\infty} a_{p} r^{n_{p}}$ is the series arising from $\lambda(r)$ when $r^{1 / \varepsilon_{p}}$ is replaced by $r^{n_{p}}$. The sum of this series is dominated by $\lambda(r)$ and from (3.2) it follows that

Now define

$$
\sum_{1}^{\infty} \frac{a_{p}}{n_{p}}=\infty
$$

$$
u\left(r e^{i \theta}\right)=\sum_{p=1}^{\infty} \frac{a_{p}}{2 K+2} r^{n_{p}} \sin \left(n_{p} \theta\right),
$$

and it follows easily that

$$
I(r, 0, \pi)=\frac{2}{2 K+2} \sum_{p=1}^{\infty} \frac{a_{p}}{n_{p}} r^{n_{p}} \rightarrow \infty \quad(r \rightarrow 1),
$$

by (3.3). By the above we see also that

$$
\left|u\left(r e^{i \theta}\right)\right| \leqq \frac{\lambda(r)}{2 K+2} \leqq k(r) \quad(0 \leqq r<1) .
$$


Remark. One might consider how much the regularity condition on $k(r)$ in Theorem 2 can be weakened. The above argument can be modified without too much difficulty to show that it is sufficient that

$$
\frac{k(R)}{k(r)} \leqq \exp \left(\alpha \cdot\left(\frac{1-r}{1-R}\right)\right) \quad(0 \leqq r<R<1),
$$

for some $\alpha$ with $0<\alpha<1$.

\section{Proof of Theorem 3}

The $k(r)$ of Theorem 3 is a function of Besicovitch. We are indebted to Siobhan Vernon for our knowledge of this function. The definition and relevant properties of this function are given in the next lemma.

Lemma 4.1 [1]. Let $\alpha_{0}=0, \alpha_{k}=1-2^{-2^{k}}(k=1,2, \ldots)$ and define $f(r)$ for $0 \leqq r<1$ by:

$$
f(0)=1, f\left(\alpha_{k}\right)=2^{2^{k-1}}, \quad f \text { is linear on }\left[\alpha_{k}, \alpha_{k+1}\right] \quad(k=0,1,2, \ldots) .
$$

Then $\int_{0}^{1} f(r) d r=\infty$, but if $P(r)$ is a power series with non-negative coefficients and $P(r) \leqq f(r)(0 \leqq r<1)$, then

$$
\int_{0}^{1} P(r) d r \leqq C,
$$

where $C$ is some fixed positive constant.

In what follows $f$ will always denote the function of Besicovitch. Let $u(z)$ be harmonic in $U$ with $u(0)=0$ and suppose that

$$
|u(z)| \leqq f(r) \quad(|z| \leqq r),
$$

and let $g=u+i v$, as in $\S 1$. As in the proof of Theorem 1, we have

$$
\left|g^{\prime}\left(r e^{i \theta}\right)\right| \leqq \frac{8 f((1+r) / 2)}{1-r} \quad(0 \leqq r<1) .
$$

From the proof of Theorem 1 it is clear that in order to prove Theorem 3 it is sufficient to show that, for some fixed constant $\gamma$ depending only on the constant $C$ in Lemma 4.1,

$$
\int_{0}^{1}\left|g\left(r e^{i \theta}\right)\right| d r \leqq \gamma \quad(0 \leqq \theta \leqq 2 \pi) .
$$

Without loss of generality assume that $\theta=0$. It is easy to show that

$$
\int_{0}^{r}|g(t)| d t \leqq \int_{0}^{r}(1-s)\left|g^{\prime}(s)\right| d s
$$


and what we now prove is that

$$
\int_{0}^{1}(1-r)\left|g^{\prime}(r)\right| d r \leqq \gamma .
$$

Let $D$ be the subdomain of $U$ bounded by segments of the lines from $z=1$ making angles $\pm 3 \pi / 4$ with the positive real axis and the major arc of the tangential circle $\{|z|=1 / \sqrt{2}\}$. Suppose that $\zeta=\varphi(z)$ maps $D$ onto the unit $\zeta$-disc $V$ with $\varphi(0)=0, \varphi(1)=1$ and real $z$ mapping into real $\zeta$. Let $z=\psi(\zeta)$ be the inverse of $\varphi$ and in $V$ set

$$
G(\zeta)=(1-\psi(\zeta)) g^{\prime}(\psi(\zeta))
$$

If one applies a conformal map to $D$ which opens the angle $\pi / 2$ at $z=1$ to an angle $\pi$ and considers the map of $V$ onto this latter domain we see that it is a convex mapping and maps $\{|\zeta|=\varrho\}$ onto a convex curve. When we relate this to the mapping $\psi$ of $V$ onto $D$ we see that for $\varrho$ near to 1 at any rate the point on the image of $\{|\zeta|=\varrho\}$ farthest from the origin is $\psi(\varrho)=r$, say. An elementary argument shows that for some positive constants $\sigma_{1}, \sigma_{2}$ such $\varrho, r$ near 1 are connected by

$$
1-\sigma_{2} \sqrt{1-\varrho} \leqq r \leqq 1-\sigma_{1} \sqrt{1-\varrho} .
$$

From (4.1), (4.3), the fact that $D$ subtends a Stolz angle at $z=1$ and the above it follows that for some constant $x$,

$$
|G(\zeta)| \leqq \chi f\left(1-\sigma_{1} \sqrt{1-\varrho}\right) . \quad(|\zeta|=\varrho) .
$$

We now quote a result of Erdős and Kővári which we require.

Lemma 4.2 [3]. If $h(z)$ is an entire function and $M(r, h)=\max _{|z|=r}|h(z)|$, then there exists a power series $P(r)$ with non-negative coefficients such that

$$
\frac{1}{6}<\frac{M(r, h)}{P(r)}<3 \quad(r \geqq 0) .
$$

Corollary. The result of the lemma remains true when $h(z)$ is analytic in $U$ and $M(r, h) / P(r)$ is considered in $0 \leqq r<1$.

The corollary follows by the same argument as the lemma itself, mutatis mutandis. Though the corollary is more convenient for our arguments the result of the lemma itself could be used instead and so the validity of our results is not dependent upon the corollary, whose proof is omitted.

Choose a power series $P(\varrho)$ with non-negative coefficients so that

$$
\frac{1}{6}<\frac{M(\varrho, G)}{P(\varrho)}<3 \quad(0 \leqq \varrho<1) .
$$

From (4.4),

$$
M(\varrho, G)<3 P(\varrho)<18 M(\varrho, G) \leqq 18 \varkappa f\left(1-\sigma_{1} \sqrt{1-\varrho}\right) .
$$


Define $\varrho_{k}$ by $\alpha_{k}=1-\sigma_{1} \sqrt{1-\varrho_{k}}$, i.e. $1-\varrho_{k}=\left(\left(1-\alpha_{k}\right) / \sigma_{1}\right)^{2}$, where the $\alpha_{k}$ are given in Lemma 4.1. We now follow the argument of Besicovitch in [1] to show that

$$
\int_{0}^{1} \frac{P(\varrho)}{\sqrt{1-\varrho}} d \varrho \leqq \text { const. }
$$

Suppose that $P(\varrho)=\sum_{0}^{\infty} b_{n} \varrho^{n}$ and write

$$
\int_{\varrho_{k}}^{e_{k+1}} \frac{P(\varrho)}{\sqrt{1-\varrho}} d \varrho=\int_{\varrho_{k}}^{\varrho_{k+1}} \frac{\left(\sum_{0}^{M} b_{n} \varrho^{n}+\sum_{M+1}^{\infty} b_{n} \varrho^{n}\right)}{\sqrt{1-\varrho}} d \varrho,
$$

where $M$ is a positive integer to be specified later. We have that

and that

$$
\begin{aligned}
& \int_{\varrho_{k}}^{e_{k+1}} \frac{\sum_{0}^{M} b_{n} \varrho^{n}}{\sqrt{1-\varrho}} d \varrho \leqq \sum_{0}^{M} b_{n} \varrho_{k}^{n} \int_{\varrho_{k}}^{\varrho_{k+1}}\left(\frac{\varrho}{\varrho_{k}}\right)^{M} \frac{d \varrho}{\sqrt{1-\varrho}} \\
& \leqq 18 x f\left(1-\sigma_{1} \sqrt{1-\varrho_{k}}\right) \int_{\varrho_{k}}^{e_{k+1}}\left(\frac{\varrho}{\varrho_{k}}\right)^{M} \frac{d \varrho}{\sqrt{1-\varrho}} ;
\end{aligned}
$$

Now,

$$
\begin{aligned}
& \int_{\ell_{k}}^{e_{k+1}} \frac{\sum_{M+1}^{\infty} b_{n} \varrho^{n}}{\sqrt{1-\varrho}} d \varrho \leqq \sum_{M+1}^{\infty} b_{n} \varrho_{k+1}^{n} \int_{\varrho_{k}}^{\varrho_{k+1}}\left(\frac{\varrho}{\varrho_{k+1}}\right)^{M} \frac{d \varrho}{\sqrt{1-\varrho}} \\
& \leqq 18 x f\left(1-\sigma_{1} \sqrt{1-\varrho_{k+1}}\right) \int_{\varrho_{k}}^{\varrho_{k+1}}\left(\frac{\varrho}{\varrho_{k+1}}\right)^{M} \frac{d \varrho}{\sqrt{1-\varrho}} .
\end{aligned}
$$

$$
\int_{\varrho_{k}}^{\varrho_{k+1}} \frac{\varrho^{M}}{\sqrt{1-\varrho}} d \varrho=\int_{\varrho_{k}}^{\varrho_{k+1}} \frac{e^{M \log (1-(1-\varrho))}}{\sqrt{1-\varrho}} d \varrho \leqq \int_{\varrho_{k}}^{\varrho_{k+1}} \frac{e^{-M(1-\varrho)}}{\sqrt{1-\varrho}} d \varrho
$$

and if we put $M(1-\varrho)=t$ the last integral becomes

$$
\int_{M\left(1-e_{k+1}\right)}^{M\left(1-e_{k}\right)} \frac{e^{-t}}{\sqrt{t / M}} \frac{d t}{M} \leqq \frac{1}{\sqrt{M}} \int_{0}^{\infty} \frac{e^{-t}}{\sqrt{t}} d t .
$$

Hence, for large $k$, writing $\sigma$ for $\sigma_{1}$,

$$
\begin{aligned}
\int_{\varrho_{k}}^{\varrho_{k+1}} \frac{P(\varrho)}{\sqrt{1-\varrho}} d \varrho & \leqq \text { const. } \frac{1}{\sqrt{M}}\left\{f\left(\alpha_{k}\right) \varrho_{k}^{-M}+f\left(\alpha_{k+1}\right) \varrho_{k+1}^{-M}\right\} \\
& \leqq \text { const. } \frac{1}{\sqrt{M}}\left\{2^{2^{k-1}} e^{2 M\left(1-\varrho_{k}\right)}+2^{2^{k}} e^{2 M\left(1-\varrho_{k+1}\right)}\right\} \\
& =\text { const. } \frac{1}{\sqrt{M}}\left\{2^{2^{k-1}} e^{\left(2 M / \sigma^{2}\right) 2^{-2^{k+1}}}+2^{2^{k}} e^{\left(2 M / \sigma^{2}\right) 2^{-2^{k+2}}}\right\} .
\end{aligned}
$$


Now choose $M=2^{2^{k+1}} k^{4}$ so that the right hand side above is bounded by

$$
\text { const. } \frac{2^{-2^{k}}}{k^{2}}\left\{2^{2^{k-1}} e^{2 k^{4} / \sigma^{2}}+2^{2^{k}} e^{\left(2 / \sigma^{2}\right) 2^{-2^{k+1}} k^{4}}\right\} \leqq \frac{\text { const }}{k^{2}} .
$$

Hence (4.5) follows and consequently

$$
\int_{0}^{1} \frac{M(\varrho, G)}{\sqrt{1-\varrho}} d \varrho \leqq \text { const. }
$$

With $r=\psi(\varrho)$ we observed earlier that if $r, \varrho$ are near to 1 , then $1-\sigma_{2} \sqrt{1-\varrho} \leqq r$ and from

$$
(1-r)\left|g^{\prime}(r)\right| \leqq M(\varrho, G)
$$

it follows that for some fixed $\eta(0<\eta<1)$,

$$
\int_{\eta}^{1}(1-r)\left|g^{\prime}(r)\right| d r \leqq \int_{\eta}^{1} M\left(1-\frac{(1-r)^{2}}{\sigma_{2}^{2}}, G\right) d r .
$$

If we now set $t=1-(1-r)^{2} / \sigma_{2}^{2}$ we obtain, for some $\eta^{\prime}$ that

$$
\int_{\eta}^{1}(1-r)\left|g^{\prime}(r)\right| d r \leqq \text { const. } \int_{\eta^{\prime}}^{1} \frac{M(t, G)}{\sqrt{1-t}} d t
$$

and so (4.2) follows.

\section{Concluding remark}

Theorem 3 has a bearing on the 'best' regularity condition on $k(r)$ in Theorem 2 . Though the condition considered at the end of $\$ 3$ seems very weak indeed, the Besicovitch function in $\S 4$ does not satisfy such a condition for any $\alpha$.

\section{References}

[1] Besicovitch, A. S.: Two problems on power series. - J. London Math. Soc. 38, 1963, 223-225.

[2] Duren, P. L.: Theory of $H^{p}$ spaces. Pure and Applied Mathematics. Vol. 38. - Academic Press, New York-London, 1970.

[3] Erdős, P., and T. KővÁrI: On the maximum modulus of entire functions. - Acta Math. Acad. Sci. Hungar. 7, 1956, 305-317. (Russian summary.)

[4] Hayman, W. K., and B. Korenblum: An extension of the Riesz-Herglotz formula. - Ann. Acad. Sci. Fenn. Ser. A I Math. 2, 1976, 175-201.

[5] Spaces of analytic functions, Kristiansand, Norway 1975, edited by O. B. Bekken, B. K. Øksendal, and A. Stray. - Lecture Notes in Mathematics 512, Springer-Verlag, Berlin-Heidelberg-New York, 1976.

The Open University

Faculty of Mathematics

Milton Keynes

England

Received 29 March 1983 